\title{
Das Qualitätshaus als Instrument zur Leistungsverbesserung von Studienzentralen der Pädiatrischen Onkologie und Hämatologie
}

\author{
U. Creutzig ${ }^{1}$ \\ J. Hannemann ${ }^{2}$ \\ I. Krämer ${ }^{2}$ \\ M. Zimmermann² \\ R. Herold ${ }^{1}$ \\ J. F. Marx ${ }^{3}$
}

\author{
The "Quality House Pediatric Oncology" as an Instrument for Improving \\ the Performance of the Trial Centers
}

\section{Zusammenfassung}

Im Rahmen des Arbeitsprojektes „Studienunterstützung“ des Kompetenznetzes Pädiatrische Onkologie und Hämatologie $(\mathrm{KPOH})$ werden Therapieoptimierungsstudien der Gesellschaft für Pädiatrische Onkologie und Hämatologie unterstützt, um den steigenden rechtlichen, ethischen und administrativen Anforderungen gerecht zu werden. Durch diese qualitätsverbessernden Maßnahmen werden die Aussagen der Studien überprüfbarer und sicherer, die Durchführung dieser Studien aber aufwendiger. Als zentrales Instrument der Studienunterstützung wird ein Qualitätshaus zur Leistungsverbesserung der entsprechenden Studienzentralen aufgebaut. Es trägt dazu bei, die Anforderungen an ein studienbezogenes, systematisches Qualitätsmanagement umzusetzen. Das „Qualitätshaus der Pädiatrischen Onkologie und Hämatologie“ umfasst eine Tätigkeitsbeschreibung aller an einer Studie beteiligten Personen. Seine Prozesslandkarte beschreibt die vielfältigen Arbeitsabläufe zur Planung und Durchführung einer klinischen Studie. Die in der Landkarte dargestellten Wertschöpfungsprozesse werden Schritt für Schritt erklärt und die einzelnen Aufgaben den zuständigen Personen zugeordnet. $\mathrm{Zu}$ jedem Prozessschritt werden zudem erläuternde Texte und ggf. notwendige Arbeitshilfen (Standard Operating Procedures und andere Dokumente) hinterlegt. Ebenso werden Verbesserungsmöglichkeiten mit direktem Bezug zum Arbeitsablauf aufgezeigt. Dieses vollständig elektronisch unterstützte System wird schrittweise weiter ausgebaut und im

\section{Abstract}

The project group "Central Trial Support" of the German Competence Network Pediatric Oncology and Haematology supports the members of the Society of Pediatric Oncology and Haematology in their effort to cope with the growing statutory, ethical and administrative requirements for therapy optimization studies (investigator-initiated, non-profit clinical trials). By these quality improvement measures the studies will become more revisable and reliable, but at the same time their processing will become more and more complex. The basic instrument of the project group "Central Trial Support" will be the so-called "Quality House" which has been built up in order to improve the performance of the associated study centres and to help put a systematic quality management system into practice. The "Quality House Pediatric Oncology" comprises detailed descriptions of the activities of all trial center co-workers. Its process map details all operational sequences which constitute an efficiently performing trial center. The so-called value adding processes are explained step by step, and the associated specific tasks are assigned to each respective co-worker. At each process step, the person in charge will have explanatory descriptions at her/his disposal and - if necessary - further problem solving means as well as references to possible optimization measures (e.g. Standard Operating Procedures and other documents). The German Competence Network Pediatric Oncology and Haematology will be implementing this electronic quality management system in

Institutsangaben

${ }^{1}$ Koordinationszentrale KPOH, Hannover und Klinik für Pädiatrie m. S. Hämatologie/Onkologie,

Campus-Virchow-Klinikum, Charité - Universitätsmedizin Berlin, für das Kompetenznetz

Pädiatrische Onkologie und Hämatologie (KPOH)

${ }^{2}$ Koordinationszentrale KPOH, Projekt Studienunterstützung. Medizinische Hochschule Hannover/

Kinderheilkunde, Pädiatrische Hämatologie/Onkologie

${ }^{3}$ BeraCon Unternehmensentwicklung, Köln

Korrespondenzadresse

Prof. Dr. med. Ursula Creutzig · Koordinationszentrale Kompetenznetz Pädiatrische Onkologie und Hämatologie (KPOH), Geschäftsführung · Universitätsklinikum Münster · Klinik und Poliklinik für

Kinder- und Jugendmedizin · Pädiatrische Hämatologie und Onkologie · Albert-Schweitzer-Str. 33 . 48129 Münster·Tel.: 0511/6046677·Fax: 0511/6046404·E-mail: ursula@creutzig.de

Bibliografie

Klin Pädiatr 2005; 217: 114-119 • @ Georg Thieme Verlag KG Stuttgart · New York

DOI 10.1055/s-2005-836507

ISSN 0300-8630 
Informationsportal den Studienzentralen zur Verfügung gestellt. Dadurch wird eine Qualitätssteigerung ihrer Arbeit und eine Anerkennung der gewährleisteten Qualitätsverpflichtung durch Förderer und Behörden möglich.

\section{Schlüisselwörter}

Qualitätsmanagement · klinische Studie · Pädiatrische Onkologie und Hämatologie trial centers which will convince both sponsors and authorities of the compliance with requirements and standards.

\section{Key words}

Quality management · clinical trials · pediatric oncology and haematology

\section{Einleitung}

Eine der zentralen Aufgaben des Kompetenznetzes Pädiatrische Onkologie und Hämatologie (KPOH; Sprecher: Prof. Dr. G. Henze, Berlin) betrifft die Unterstützung von Therapieoptimierungsstudien (TOS), die im Rahmen der Gesellschaft für Pädiatrische Onkologie und Hämatologie (GPOH) durchgeführt werden.

Das wesentliche Ziel dieser Maßnahme ist die Verbesserung der Qualität der Studiendurchführung und die Einführung eines Qualitätsmanagementsystems, das in jeder Einzelnen dieser Studien zur Anwendung kommen kann.

Die Notwendigkeit eines Qualitätsmanagements nimmt im Gesundheitswesen aufgrund gesetzlicher Forderungen, aber auch aus der Einsicht, dass damit begrenzte Ressourcen effizienter eingesetzt werden können, generell zu. Auf gesetzlicher Seite sind für Studienzentralen das kürzlich reformierte Arzneimittelgesetz [6] sowie die „Verordnung über die Anwendung der Guten Klinischen Praxis bei der Durchführung von klinischen Prüfungen mit Arzneimitteln zur Anwendung am Menschen“ (GCP-V) [5] zu nennen, welche die Liste externer Anforderungen deutlich verlängert und den Regelungsbedarf weiter erhöht haben. In der Vergangenheit kamen im Gesundheitswesen alle Arten von in der Industrie seit vielen Jahren bewährten Instrumenten aus dem Qualitätsmanagement zum Einsatz. Hier sind beispielhaft die internationale Qualitätsnorm ISO 9001:2000 [7] und der auf einem europäischen Qualitätswettbewerb basierende EFQM (European Foundation for Quality Management)-Ansatz [11] zu nennen. Weiterhin werden verschiedene Derivate entwickelt, wie zum Beispiel KTQ (Kooperation für Transparenz und Qualität im Krankenhaus), welches den Ansatz der EFQM auf Krankenhäuser überträgt.

Der in diesem Artikel geschilderte methodische Ansatz zeigt, wie die Anforderungen an eine Studienzentrale im Sinne eines systematischen Qualitätsmanagements umgesetzt werden.

\section{Anforderungen an eine Studienzentrale}

Im Rahmen von Studiengruppen, die teilweise schon in den 70erJahren gegründet wurden, werden derzeit 27 bundesweite Therapieoptimierungsstudien im Bereich der Pädiatrischen Onkologie und Hämatologie durchgeführt. Kontinuierlich werden neue Studienprotokolle, aufbauend auf den vorangehenden, herausgegeben.

Über 90\% aller an Krebs erkrankten Kinder und Jugendlichen in Deutschland werden nach diesen einheitlichen Therapievor- gaben behandelt [9]. Die Therapie wird größtenteils risikoadaptiert durchgeführt und eine Weiterentwicklung wird u.a. durch die randomisierte Prüfung neuer Therapieoptionen möglich. Bei den im Kindesalter z.T. sehr seltenen Erkrankungen ist häufig nur der historische Vergleich zur Effizienzbeurteilung möglich, da das Erreichen ausreichender Fallzahlen für randomisierte Vergleiche zu viel Zeit erfordern würde.

Die ständige Verbesserung der Behandlung hat zu neuen, weiterführenden wissenschaftlichen Erkenntnissen geführt und im Ergebnis die langfristige Überlebenswahrscheinlichkeit auf mehr als 75\% für die Gesamtgruppe dieser Patienten erhöht [3].

Die Aufgaben, die im Rahmen der Studiendurchführung von Seiten der Studienzentralen und der beteiligten Kliniken zu bewältigen sind, sind vielseitig und sehr komplex. Erschwerend kommt hinzu, dass bundesweit häufig wechselnde Personen unterschiedlicher Qualifikation beteiligt sind (z.B. Einsatz von Rotationsärzten sowohl in Studienzentralen als auch in den Behandlungszentren). Dies unterstreicht die Bedeutung einer schnellen, qualifizierten Einarbeitung neuer Mitarbeiter sowie die Notwendigkeit einer klaren Aufgabenverteilung.

Hinzu kommt, dass inzwischen die Anzahl der gesetzlichen Vorgaben deutlich angestiegen ist (aktuelle Übersicht z.B. in [2]). Neben der fachlichen Qualifikation wird von den handelnden Mitarbeitern die Erfüllung dieser Vorgaben erwartet. Um hier ein Höchstmaß an Rechtssicherheit zu gewährleisten, ist es erforderlich, einheitliche Vorgehensweisen, z.B. bei der Meldung von „Serious Adverse Events“(SAE), festzulegen.

Zusätzlich werden Musterlösungen und Vorlagen benötigt, die die Erstellung von Studienprotokollen und zugehörigen Dokumenten erleichtern. So können u.a. Inhaltsverzeichnis und Textbausteine für die Erstellung von Studienprotokollen in Form eines „Masterprotokolls“ zur Verfügung gestellt werden. In ein zentrales Dokument können die Erfahrungen zurückfließen, die mit einzelnen Anträgen gemacht wurden, um somit eine kontinuierliche Verbesserung der Arbeitsergebnisse zu gewährleisten. Gleichzeitig stellen die Musterlösungen sicher, dass die umfangreichen Anforderungen an Bewertung und Dokumentation erfüllt werden.

Die Anforderungen an die klinischen Studien in der Pädiatrischen Onkologie und Hämatologie decken sich in vielen Punkten mit den zentralen Anliegen normenorientierter Qualitätsmanagementsysteme. Daher erscheint es sinnvoll, die im Qualitätsmanagement entwickelten Instrumente daraufhin zu überprüfen, ob sie sich für die Erfüllung der Aufgaben von Studienzentralen eignen. In diesem Zusammenhang werden hier auch Begriffe 


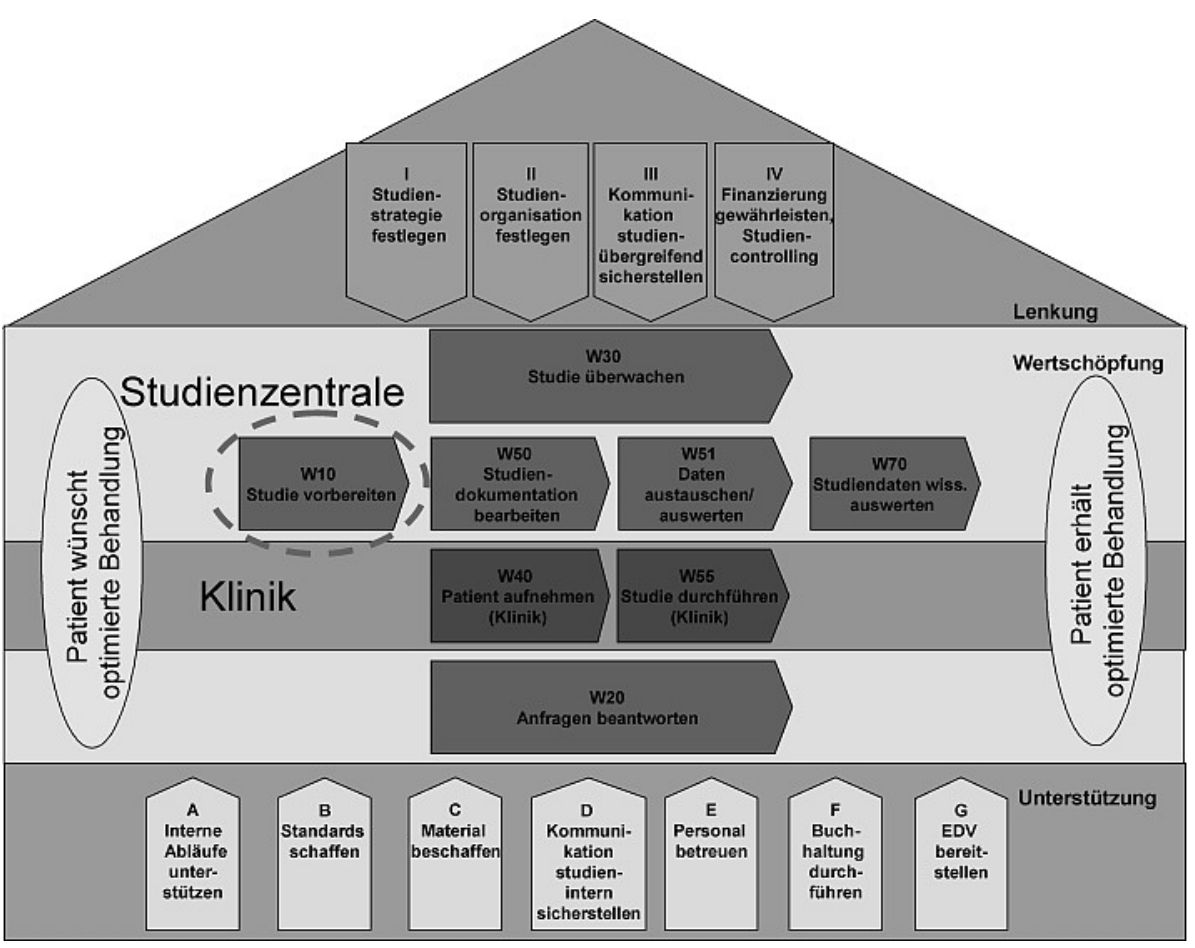

Abb. 1 Das Qualitätshaus Pädiatrische Onkologie und Hämatologie.

verwendet (z.B. „Kundengruppen“), die aus dem Sprachgebrauch des Qualitätsmanagements stammen und im medizinischen Umfeld bislang nicht gebräuchlich sind.

\section{Ein prozessorientierter Lösungsweg: das Qualitätshaus}

Mit der ISO 9001:2000 verbreitet sich im Qualitätsmanagement der prozessorientierte Ansatz. Prozessorientiert bedeutet, dass die Anforderungen an eine Organisation nicht mehr abstrakt nach Elementen gegliedert in einem Handbuch aufgelistet werden, sondern dass die Basis eine transparente Darstellung aller wesentlichen Abläufe (Prozesse) in der Organisation ist. Keineswegs führt jedoch die stoische Abarbeitung der Forderungen dieser bewährten Norm zum Ziel, sondern nur die kritische Diskussion um jede Anforderung und die Klärung, wie die einzelnen Anforderungen nutzbringend für die Organisation umgesetzt werden können. Da die Zertifizierung bei Studienzentralen nicht im Vordergrund steht, konnte die Norm unvoreingenommen als Ideensammlung zur Verbesserung der Qualität genutzt werden.

Das Projekt „Studienunterstützung“ des KPOH begann die Erarbeitung eines Qualitätssicherungskonzepts 2002 und wurde dabei in den Jahren 2003 und 2004 von der BeraCon Unternehmensentwicklung kontinuierlich betreut. Die Aufgabe der Unternehmensberatung liegt darin, die Erfahrung zum Qualitätsmanagement aus zahlreichen anderen Organisationen und Unternehmen für die Studien der GPOH nutzbar zu machen und sie durch „Coaching“ bei der Einhaltung der gesteckten Ziele zu unterstützen. Ebenfalls im Jahr 2003 wurde eine Arbeitsgruppe aus Mitarbeitern verschiedener Studienzentralen gegründet, die über die von der Studienunterstützung erarbeiteten strukturellen Vorschläge berät und mit dieser gemeinsam verabschiedet.
Der erste Schritt des Projekts im Hinblick auf eine Verbesserung der Qualität in Studienzentralen bestand darin, die Ziele und Abläufe der „GPOH-Studien“ [10] zu formulieren und daraus eine Prozesslandkarte abzuleiten. Als Ergebnis entstand daraus das „Qualitätshaus Pädiatrische Onkologie und Hämatologie“, welches die Tätigkeiten aller an einer Studie Beteiligten in einen ordnenden Rahmen stellt. Die Prozesslandkarte fasst alle Prozesse zusammen, die direkt oder indirekt zur Erbringung der gewünschten Leistung beitragen (Abb.1).

Die zentrale Frage des zweiten Schritts des Projekts bestand in der Feststellung, welche „Kundengruppen“ in welcher Form im Qualitätshaus berücksichtigt werden sollen. Die Differenzierung nach Patienten, den behandelnden Kliniken und den Studienzentralen ging ebenfalls in die Prozesslandkarte ein. Abb. 1 zeigt den aktuellen Stand, wobei sich die Prozesslandkarte mit zunehmender Durchdringung und wechselnden Anforderungen aus dem Umfeld verändert.

Das Qualitätshaus besteht aus mehreren horizontal angeordneten Teilbereichen. Die Mitte des Hauses wird von den Wertschöpfungsprozessen gebildet. In den Wertschöpfungsprozessen werden die Schritte beschrieben, die zur Erreichung des vorher festgelegten Zieles notwendig sind. So ist beispielsweise das wesentliche Ziel der Arbeit einer Studienzentrale, eine optimierte Behandlung des Patienten zu gewährleisten.

Neben den eigentlichen Wertschöpfungsprozessen bilden die Unterstützungsprozesse das Fundament des Qualitätshauses. Hier sind Abläufe beschrieben, die zur Erbringung der Wertschöpfung erforderlich sind, wie z.B. Personalbetreuung, EDV und Bereitstellung von Standards.

Das Dach des Qualitätshauses bilden die Lenkungsprozesse, die übergeordnete Aufgaben erfüllen, wie die Festlegung der Strate- 


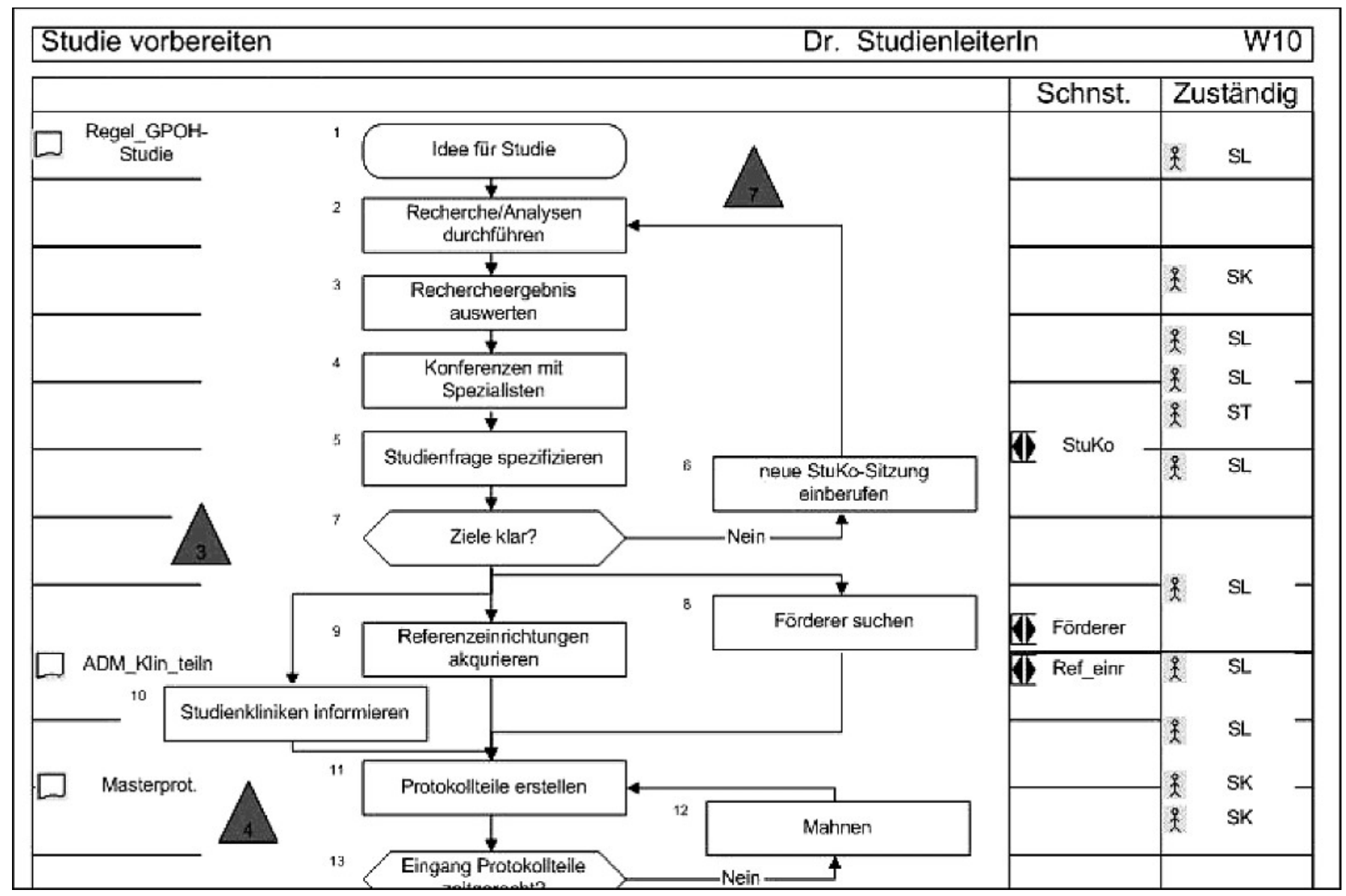

Abb. 2 Ausschnitt aus Prozessablauf W10 „Studie vorbereiten“.

\section{SAE-Meldeverpflichtungen bei klinischen Prüfungen}

Zu beachtende Dokumente:

- 12. Gesetz zur Änderung des AMG (veröffentlicht im Bundesanzeiger am 05.08.2004)

- GCP-Verordnung (veröffentlicht im Bundesanzeiger am 12.08.2004)

- Detailed guidance on the collection, verification and presentation of adverse reaction reports arising from clinical trials on medicinal products for human use (Stand April 2004)

Verwendete Abkürzungen:

BOB zuständige Bundesoberbehörde

EK zuständige Ethikkommission

EU zuständige Behörden an der klinischen Prüfung beteiligter EU-Mitgliedsstaaten

lok. Beh. zuständige (lokale) Behörde

Prüfer alle an der Prüfung beteiligte Prüfer

NW Nebenwirkung

Personenbezogene Daten sind vor der Übermittlung zu pseudonymisieren!

SAE-Meldeverpflichtungen des Sponsors während der Laufzeit der klinischen Prüfung ( $\$ 13 \mathrm{GCP}-\mathrm{V}$ )

\begin{tabular}{|c|c|c|c|c|c|c|c|}
\hline Art & Zeit & Was? & вов & EK & EU & $\begin{array}{l}\text { lok. } \\
\text { Beh. }\end{array}$ & Prüfer \\
\hline unerwünschte Ereignisse & auf Anforderung & $\begin{array}{l}\text { ausführliche Dokumentation aller } \\
\text { gemeldeten Ereignisse }\end{array}$ & $\mathbf{x}$ & & $\mathbf{x}$ & & \\
\hline $\begin{array}{l}\text { Verdachtsfall unerwartete } \\
\text { schwerwiegende Nebenwirkung } \\
\text { (Tod, lebensbedrohlich) }\end{array}$ & $\begin{array}{l}\text { unverzüglich (spätestens } 7 \text { Tage } \\
\text { nach Bekanntwerden) }\end{array}$ & für die Bewertung wichtige Informationen & $\mathbf{x}$ & $\mathbf{x}$ & $\mathbf{x}$ & & $\mathbf{x}$ \\
\hline $\begin{array}{l}\rightarrow \text { Folgemeldung mit weiteren } \\
\text { wichtigen Informationen }\end{array}$ & innerhalb max. 8 weiteren Tagen & für die Bewertung wichtige Informationen & $\mathbf{x}$ & $\mathbf{x}$ & $\mathbf{x}$ & & $\mathbf{x}$ \\
\hline
\end{tabular}

Abb. 3 Beispiel für eine Arbeitshilfe. 


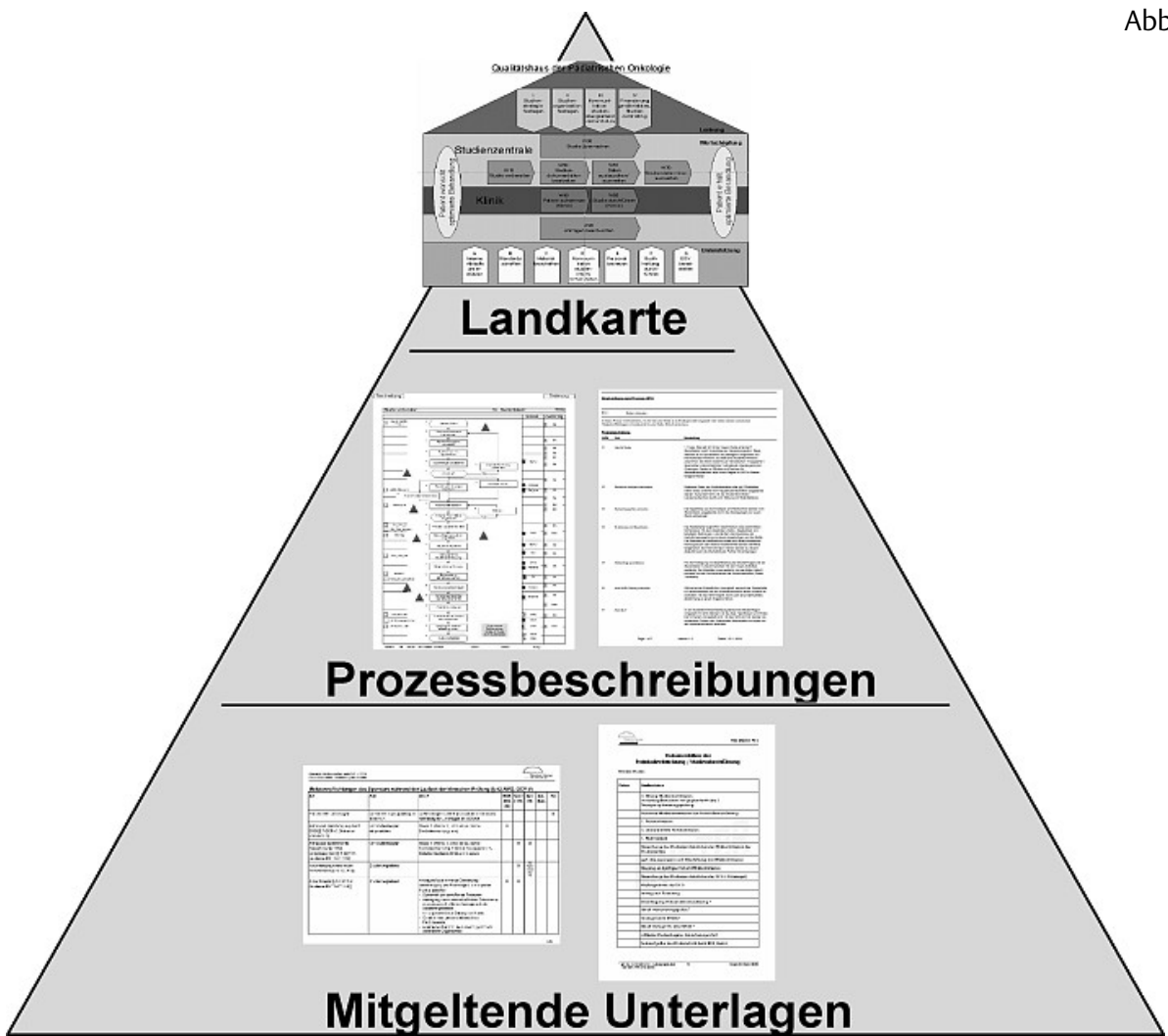

Abb. 4 Die verschiedenen Ebenen des Systems.

gie, organisatorische Grundsatzentscheidungen und Fragen der Finanzierung.

Die Umsetzung des Qualitätshauses erfolgt mit der Software BeraCon Process2002 [1]. Mit dieser Software können die Strukturen visuell dargestellt werden, gleichzeitig liegen alle Informationen datenbankgebunden vor. Die Ergebnisse werden zugriffsgeschützt im Informationsportal den Mitarbeitern der GPOHStudienzentralen zur Verfügung gestellt. Auf diese Weise gibt es eine zentrale Dokumentation, die die „Best Practice“-Erfahrungen bei der Vorbereitung, Durchführung und Nachbereitung von Studien beschreibt.

Jeder der Wertschöpfungsprozesse wird als Ablauf beschrieben. Beispielhaft wird in Abb. 2 der Prozess „W10“ gezeigt, der in der Prozesslandkarte (Abb.1) rot eingekreist ist.

Der Ablauf wird ausgehend von einem Startereignis Schritt für Schritt erklärt, wobei jedem Prozessschritt in der Spalte „Zuständig“ eine verantwortliche Funktion zugeordnet wird (z.B. SK = Studienkoordinator, SL=Studienleiter). Diese Information wird dazu genutzt, um hieraus automatisch vollständige Beschreibungen der Funktionen zu erzeugen.

Jedem Prozessschritt ist ein erläuternder Text zugeordnet, der als Dokumentation abrufbar ist. Falls erforderlich, werden in der linken Spalte einzelnen Prozessschritten Dokumente, z.B. spezielle Arbeitshilfen, zugeordnet, die Lösungsansätze liefern oder beschreiben, wie einzelne Tätigkeiten durchzuführen sind. Hier finden auch die „Standard Operating Procedures“(SOP, s.u.) ihren Platz. Verfügbare Dokumente sind einem entsprechenden Symbol hinterlegt und jederzeit aus dem Prozess aufrufbar. Zu- sätzlich besteht die Möglichkeit, diese Dokumente aus einer Dokumentenliste heraus aufzurufen.

Durch verschiedenfarbige Dreiecke werden Verbesserungsmöglichkeiten mit direktem Bezug zum Ablauf festgehalten. Zu einem späteren Zeitpunkt kann darüber entschieden werden, ob und in welcher Form auf diese Schwachstellen eingegangen werden kann. Hierbei findet grundsätzlich eine Bewertung von Aufwand und erwartetem Nutzen statt, um die begrenzten Ressourcen dort einzusetzen, wo der größte Effekt erzielt werden kann. Durch die Umsetzung von Korrekturmaßnahmen werden Schwachstellen nach und nach beseitigt und die Prozessabläufe bei Bedarf an neue Gegebenheiten angepasst. Die Abläufe unterliegen somit einem kontinuierlichen Verbesserungsprozess.

Das Qualitätshaus zeigt auch unerfahrenen Mitarbeitern schnell auf, welche Aufgaben wann wahrgenommen werden und wie einzelne Prozessschritte zusammenhängen.

Im nächsten Schritt entstehen Standard Operating Procedures (SOP), die verbindlich festlegen, wie einzelne Tätigkeiten durchzuführen sind, sowie weitere konkrete Hilfsmittel (Abb. 3).

Die Arbeitshilfen richten sich nach den aktuellen rechtlichen Erfordernissen, so dass deren Anwendung ein Höchstmaß an Rechtssicherheit, unter der Voraussetzung einer kontinuierlichen Pflege des Systems, gewährleistet.

Mit der zentralen Prozessdokumentation werden erforderliche oder sinnvolle Veränderungen im Ablauf nur noch an einer Stelle dokumentiert und stehen via Informationsportal unmittelbar allen Studien zur Verfügung. 
Zusammenfassend wird die Struktur des Informationssystems in Abb. 4 dargestellt.

\section{Das Qualitätshaus als kontinuierlicher Prozess}

Qualitätsmanagement ist ein kontinuierlicher Prozess. Entsprechend wurde das Projekt langfristig angelegt und zu Projektbeginn viel Wert auf eine klare Zieldefinition gelegt. Vor der eigentlichen Effizienzsteigerung („Die Dinge richtig tun.“) war die Frage der Effektivität zu beantworten („Die richtigen Dinge tun.“).

Aus dieser Systematik ergibt sich das Jahresprogramm 2005, welches gemeinsam mit der Geschäftsführung der GPOH Ende 2004 festgelegt wurde.

Nachdem die Grundlagen mit der Erstellung des Qualitätshauses gelegt sind, geht es jetzt darum, das System schrittweise auszubauen und eine breite Anwendung in den Studienzentralen zu erreichen. Die folgenden Argumente legen es nahe, dieses System in Studienzentralen zu nutzen:

- Berücksichtung aller einschlägigen rechtlichen Erfordernisse

- höhere Rechtssicherheit für die Studienleiter

- schnellere Einarbeitung von Mitarbeitern der Studienzentralen

- Nutzung umfassender Hilfsmittel, wie Trial Master File, GCPCheckliste etc.

- Schulungsangebote für Studienleiter, Koordinatoren, Dokumentare

- angestrebte Anerkennung der Qualitätsverpflichtung bei der Vergabe des Gütesiegels der DKG und bei Förderern.

Eine entscheidende Rolle kommt der zügigen Erstellung der wesentlichen Standardarbeitsanweisungen (SOP) zu. Dabei sollen, um Doppelarbeit zu vermeiden, Erfahrungen aus vergleichbaren Einrichtungen genutzt werden. Deshalb erfolgt Anfang 2005 eine umfassende Bestandsaufnahme der Maßnahmen zur Qualitätssicherung bei den Koordinierungszentren für Klinische Studien (KKS) und Kompetenznetzen (KN), welche vom KPOH zur Ermittlung von Synergiepotenzialen ausgewertet wird. Diese Bestandsaufnahme wird im Rahmen der eigens gegründeten „Arbeitsgruppe Management Klinischer Studien“ der „Telematikplattform für Medizinische Forschungsnetze“ durchgeführt [12].

Vom KPOH-Projekt „Studienunterstützung“ werden auch in Zukunft Schulungen unter kontinuierlicher Einbindung neuer Anforderungen durchgeführt und aktualisierte Hilfsmittel für eine effektive und effiziente Studienarbeit mit Hilfe des Qualitätshauses bereitgestellt. Das vorgestellte Projekt konzentriert sich auf die Arbeit in den Studienzentralen. Eine Lösung für den hohen dokumentarischen Aufwand in den kooperierenden Kliniken wurde bisher nicht gefunden [8].

Insgesamt wird mit der Einführung eines Qualitätsmanagementsystems in Studienzentralen eine Möglichkeit geschaffen, die „Studienkultur“ der Pädiatrischen Onkologie und Hämatologie den steigenden Anforderungen der Förderer und Behörden, aber ebenso auch der betroffenen Patienten und der Öffentlichkeit, anzupassen.

Mit der Zielsetzung, umfassende Maßnahmen für die Qualitätssicherung in der Pädiatrischen Onkologie und Hämatologie bereitzustellen, die über die Studienzentralen hinaus auch Referenzzentren und vor allem die medizinische Versorgung einbeziehen, werden gegenwärtig noch weitere Projekte konzipiert und begonnen [4]. Hiermit wird die Umsetzung der 12. Novelle des Arzneimittelgesetzes seitens der GPOH und des $\mathrm{KPOH}$ in bestmöglicher Weise unterstützt.

\section{Danksagung}

Das Kompetenznetz Pädiatrische Onkologie und Hämatologie wird vom Bundesministerium für Bildung und Forschung seit 1999 gefördert, Förderkennzeichen 01 GI 9958/5.

Die Firma BeraCon ist in den Jahren 2003 und 2004 Auftragnehmer der Koordinationszentrale Kompetenznetz Pädiatrische Onkologie und Hämatologie gewesen.

\section{Literatur}

${ }^{1}$ BeraCon Unternehmensentwicklung. Software für die Dokumentation und Pflege von Geschäftsprozessen: BeraCon Process2002. http://www.beracon.de 2004

2 Boos J. Klinische Forschung im Kindesalter - Konsequenzen der 12. AMG-Novelle. Die Mitteilungen von GPOH und KPOH 2004; 4-5

${ }^{3}$ Creutzig U, Henze G, Bielack S, Herold R, Kaatsch P, Klussmann JH, Graf N, Reinhardt D, Schrappe M, Zimmermann M, Jürgens H. Krebserkrankungen bei Kindern: Erfolg durch einheitliche Therapiekonzepte seit 25 Jahren. Dt Ärztebl 2003; 100: A 842 - A 852

${ }^{4}$ Creutzig U, Jürgens H, Herold R, Göbel U, Henze G. Konzepte der GPOH und des Kompetenznetzes zur Weiterentwicklung und Qualitätssicherung in der Pädiatrischen Onkologie. Klinische Pädiatrie 2004; 216: $379-383$

${ }^{5}$ Das Bundesministerium für Gesundheit und Soziale Sicherung im Einvernehmen mit dem Bundesministerium für Wirtschaft und Arbeit. GCP-V: im Bundesgesetzblatt 12. August 2004, Jahrgang 2004, Teil I, Nr. 42. http://217.160.60.235/BGBL/bgbl1f/bgbl104s2081.pdf 2004

${ }^{6}$ Der Bundestag und der Bundesrat der Bundesrepublik Deutschland. Zwölftes Gesetz zur Änderung des Arzneimittelgesetzes, Bundesgesetzblatt 5. August 2004, Jahrgang 2004, Teil I, Nr. 41. http://217. 160.60.235/BGBL/bgbl1f/bgbl104s2031.pdf 2004

${ }^{7}$ Qualitätsmanagement DIN EN $9000 \mathrm{ff}$., Dokumentensammlung. www. beuth.de 2000

${ }^{8}$ Graf N, Göbel U. Therapieoptimierungsstudien der Gesellschaft für Pädiatrische Onkologie und Hämatologie $(\mathrm{GPOH})$ und 12. Novelle des Arzneimittelgesetzes zur Umsetzung der EU-Richtlinie. Klin Pädiatr 2004; 216: 129-131

${ }^{9}$ Kaatsch P, Spix C. German Childhood Cancer Registry - Annual Report 2003 (Jahresbericht 2003 des Deutschen Kinderkrebsregisters). Buchdruckerei Johannes Krüger OHG Berlin 2004

${ }^{10}$ Reiter A, Creutzig U, Niemeyer C M, Berthold F, Göbel U, Klingebiel T. Regeln für Kooperative Multizentrische Studien der Gesellschaft für Pädiatrische Onkologie und Hämatologie (GPOH). http://www. kinderkrebsinfo.de/e2260/e2298/e4822/ 1998

11 Stichting European Foundation for Quality Management ("EFQM"). European Foundation for Quality Management. www.efqm.org 2004

12 TMF e.V. Telematikplattform für Medizinische Forschungsnetze gem. e.V. http://www.tmf-ev.de/ 2004 